\title{
Proton beam therapy followed by pembrolizumab for giant ocular surface conjunctival malignant melanoma: A case report
}

\author{
TOSHIHIKO MATSUO ${ }^{1,2}$, OSAMU YAMASAKI ${ }^{3,4}$, TAKEHIRO TANAKA $^{5}$, \\ KUNIAKI KATSUI $^{6,7}$ and TAKAHIRO WAKI ${ }^{8}$
}

\author{
${ }^{1}$ Regenerative and Reconstructive Medicine (Ophthalmology), Okayama University Graduate School of \\ Interdisciplinary Science and Engineering in Health Systems, Okayama 700-8558; ${ }^{2}$ Department of Ophthalmology, \\ Okayama University Hospital; ${ }^{3}$ Department of Dermatology, Okayama University Graduate School of Medicine, \\ Dentistry and Pharmaceutical Sciences; ${ }^{4}$ Melanoma Center, Okayama University Hospital, Okayama 700-8558; ${ }^{5}$ Department \\ of Pathology, Okayama University Graduate School of Medicine, Dentistry and Pharmaceutical Sciences, Okayama 700-8558; \\ ${ }^{6}$ Division of Radiation Oncology, Department of Radiology, Kawasaki Medical School, \\ Kurashiki, Okayama 701-0192; ${ }^{7}$ Department of Radiology, Okayama University Graduate School of Medicine, \\ Dentistry and Pharmaceutical Sciences, Okayama 700-8558; \\ ${ }^{8}$ Department of Radiology, Tsuyama Chuo Hospital, Tsuyama, Okayama 708-0841, Japan
}

Received September 15, 2021; Accepted October 27, 2021

DOI: $10.3892 / \mathrm{mco} .2021 .2445$

\begin{abstract}
The present study describes proton beam therapy as a clinical option to achieve local control of giant conjunctival melanoma in an aged person, instead of orbital exenteration. An 80-year-old woman with one-year history of left-eye injection and hemorrhage experienced rapid growth of the ocular surface black mass. At the initial visit, a black, elastic hard, hemorrhage-prone, thickened mass in the size of 30x40 mm with a presumed wide stalk covered the total area of the lid fissure on the left side. Biopsy of the mass demonstrated anomalous melanin-containing cells in fibrin and hemorrhage, which were positive for cocktail-mix antibodies against tyrosinase, melanoma antigen recognized by $\mathrm{T}$ cells- 1 and human melanoma black-45, indicative of malignant melanoma. One month after the initial visit, the patient underwent proton beam therapy at the total dose of $70.4 \mathrm{~Gy}$ (relative biological effectiveness) in 32 fractions ( 10 min each) in one and a half months. One month after the end of proton beam therapy, 3.5 months from the initial visit, the patient was found by computed tomographic scan to have multiple metastatic lesions in bilateral lung fields. With the evidence of absent $B R A F$ mutation, the patient underwent intravenous admin-
\end{abstract}

Correspondence to: Professor Toshihiko Matsuo, Regenerative and Reconstructive Medicine (Ophthalmology), Okayama University Graduate School of Interdisciplinary Science and Engineering in Health Systems, Shikata-cho 2-5-1, Okayama 700-8558, Japan E-mail: matsuot@cc.okayama-u.ac.jp

Key words: ocular surface, conjunctiva, malignant melanoma, proton beam therapy, pembrolizumab, PD-1 inhibitor, immune checkpoint inhibitor, corneal perforation istration of pembrolizumab $77.2 \mathrm{mg}$ every three weeks five times in total. Then, three months after proton beam therapy, ocular surface melanoma almost subsided and the clear cornea allowed visualization of the intraocular lens inside the eye. In three weeks, spontaneous corneal perforation was plugged with iris incarceration. The patient died suddenly of unknown cause 7.5 months from the initial visit. The local control of giant conjunctival melanoma was achieved by proton beam therapy, leading to patient's satisfaction and better quality of life. Proton beam therapy, followed by immune checkpoint inhibitors, would become the future standard of care for unresectable giant conjunctival melanoma.

\section{Introduction}

Malignant melanoma as a whole is less frequent in Asian populations including Japanese population, compared with Caucasian populations. In the field of ophthalmology, melanoma can be encountered rarely in uvea (choroid) (1), lacrimal sac (2), conjunctiva and eyelid skin $(3,4)$. Among these melanomas in ophthalmic practice, conjunctival malignant melanoma is relatively frequent in Asian populations and Caucasian populations $(3,4)$. However, no standard treatment has been established in conjunctival melanoma which is basically at very low incidence (5).

In case of cutaneous melanoma, its excision with ample margin of the surrounding normal skin is the standard of care for local control of malignancy. In contrast, excision of conjunctival melanoma with ample normal margin cannot be achieved since the conjunctiva of limited width is the essential mucosa to maintain the ocular surface and hence, the vision. Due to this anatomical limitation, it is difficult to excise conjunctival melanoma at the early phase with ample margin of the surrounding normal tissue as is the standard of care for cutaneous melanoma. At present, local recurrence is 
a common sequela to the initial excision of conjunctival melanoma. Orbital exenteration is a final surgical option to achieve the local control in the advanced stages when conjunctival melanoma shows infiltration into the surrounding tissue such as the eyelid skin and the sclera of the eyeball. It would be disastrous for patients to lose the vision of one eye abruptly by the orbital exenteration. Invasive surgery of the orbital exenteration would also put physical and psychological burdens on the aged people who are prone to develop conjunctival melanoma.

In the general belief that melanoma would not respond sufficiently to external beam radiation, radiotherapy has played a limited role only in adjunct or palliative treatment for metastatic diseases of cutaneous melanoma $(6,7)$, conjunctival melanoma (5) and head and neck mucosal melanoma $(8,9)$. Under the circumstances, proton beam therapy has been tried as a treatment option for the local control of conjunctival melanoma and has been indeed shown to be effective (5,10-15). In Japan, proton beam therapy for head and neck cancer including melanoma (16-18) has been covered by reimbursement from the national health insurance since April 2018. Within the framework of the national health insurance, immune checkpoint inhibitors and molecular target drugs such as MEK inhibitors and BRAF inhibitors can be also used in the case of unresectable and metastatic malignant melanoma, based on the genetic testing of $B R A F$ mutations in melanoma tissues $(19,20)$.

The present study dealt with an aged patient who showed giant conjunctival melanoma at the initial presentation and who decided to choose proton beam therapy as a first-line therapeutic option for the local control. Furthermore, based on no $B R A F$ mutation detected in the melanoma tissue, pembrolizumab, PD-1 immune checkpoint inhibitor $(19,20)$, was introduced as a current standard therapy toward metastatic lung lesions after the proton beam therapy.

\section{Case report}

An 80-year-old woman noticed injection and hemorrhage in the left eye one year previously and she removed the painless ocular surface scab by herself frequently. One month previously, the black mass grew out of the lid fissure rapidly and she could not close the left eye (Fig. 1A). She visited a local hospital and was referred to Okayama University Hospital. At the initial visit, the best-corrected visual acuity was 1.2 in the right eye and light perception in the left eye. The intraocular pressure in the right eye was $12 \mathrm{mmHg}$ and the optic nerve disc had glaucomatous cupping as a cup/disc ratio of 0.9 . Otherwise, the right eye had nothing notable. She had undergone cataract surgery in the left eye four years previously. She had no other medical history and took no medication. A black, elastic hard, hemorrhage-prone, thickened mass in the size of 30x40 mm with a presumed wide stalk covered the total area of the lid fissure on the left side (Fig. 1A) and the mass moved slightly with eye movement, indicative of the tumor origin on the ocular surface.

Magnetic resonance imaging showed the intact eye ball on the left side and no infiltration deeply into the orbit (Fig. 2A). Whole-body 2-[ $\left[{ }^{18} \mathrm{~F}\right]$ fluoro-2-deoxy-D-glucose positron emission tomography (FDG-PET) showed high uptake in the eyelid area with the mass on the left side (Fig. 2B, maximum
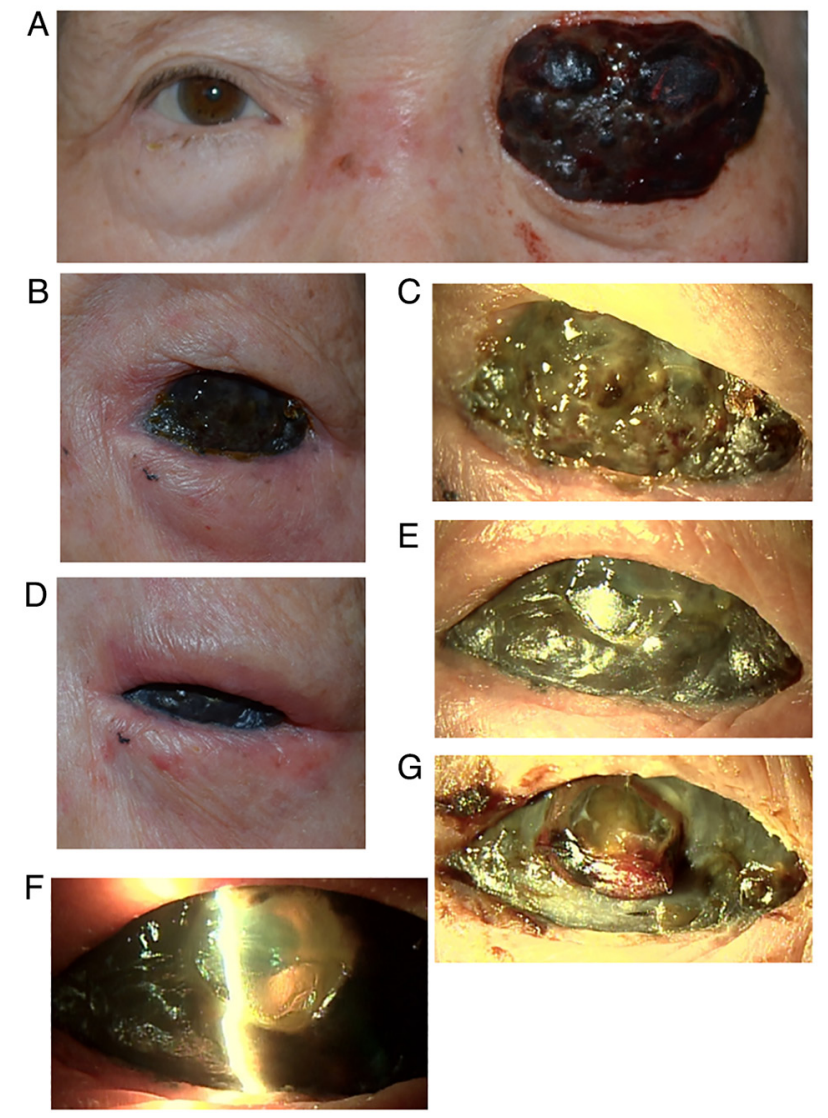

Figure 1. The mass before and after proton beam therapy. (A) A black, elastic hard, hemorrhage-prone, thickened mass arising from the ocular surface on the left side which prevents the eyelid from closing at the initial visit in an 80-year-old woman. (B and C) The mass has been reduced 2.5 months after the conclusion of proton beam therapy, 5 months from the initial visit and (D-F) has almost subsided in half a month, 5.5 months from the initial visit. (F) Note the transparent cornea to visualize inside the eye globe. (G) In three weeks, spontaneous corneal perforation has been plugged with iris incarceration.

standardized uptake value, SUVmax=14.04) and had no abnormal uptake in other sites of the body. Surface biopsy of the mass and the neutral formalin-fixed paraffin sections demonstrated anomalous melanin-containing cells in fibrin and hemorrhage (Fig. 3A). Immunostaining at the in-house pathology laboratory showed that anomalous cells were positive for cocktail-mix antibodies against tyrosinase, melanoma antigen recognized by $\mathrm{T}$ cells- 1 and human melanoma black-45 (Fig. 3B), leading to pathological diagnosis of malignant melanoma.

The patient underwent proton beam therapy one month after the initial visit, at the total dose of 70.4 Gy (relative biological effectiveness) in 32 fractions ( $10 \mathrm{~min}$ each) for one and a half months. One month after the conclusion of proton beam therapy and three and a half months from the initial visit, the patient was found by computed tomographic scan to have multiple metastatic lesions in bilateral lung fields (Fig. 2C). Excisional biopsy of residual melanotic lesions in the upper eyelid skin on the left side (Fig. 1B and D) showed melanin-containing epithelioid cells with large nuclei and apparent nucleoli (Fig. 4A and B) arranged in alveolar pattern, which confirmed the pathological diagnosis of malignant melanoma at the in-house 

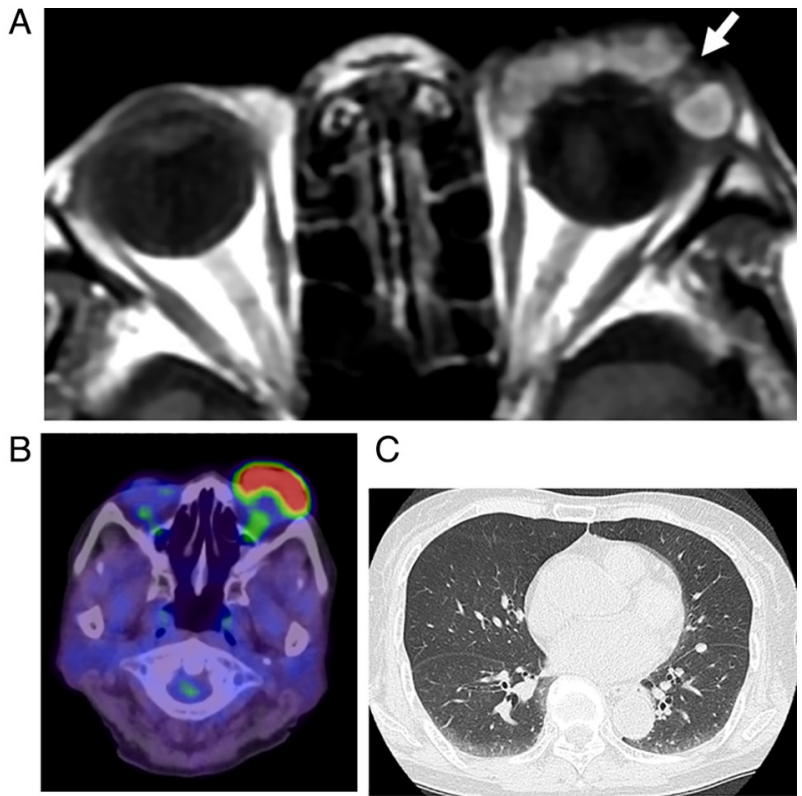

C

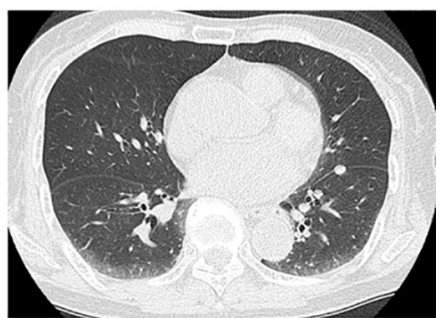

$\mathrm{D}$

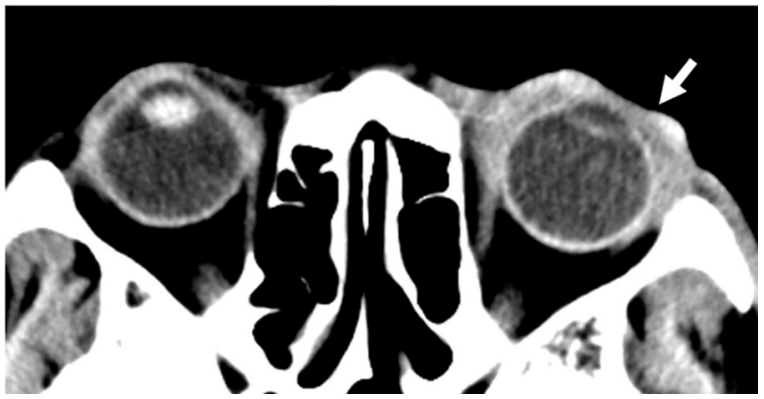

Figure 2. Images of the mass before and after proton beam therapy. (A) T1-weighted magnetic resonance image at the initial visit in an 80 -year-old woman, showing lobulated ocular surface mass with contrast enhancement on the left side (arrowed). (B) Abnormal uptake in the area of the left eyelid (maximum standardized uptake value, SUVmax=14.04) in whole-body $2-\left[{ }^{18} \mathrm{~F}\right]$ fluoro-2-deoxy-D-glucose positron emission tomography at the initial visit. (C) CT scan, showing multiple metastatic nodules in bilateral lung fields one month after the conclusion of proton beam therapy, 3.5 months from the initial visit. (D) CT scan at the same time, showing the reduced ocular surface mass with contrast enhancement (arrowed). CT, Computed tomography.

pathology laboratory. BRAF mutations (V600E and V600K) were tested by real-time PCR in the DNA sample extracted from the neutral formalin-fixed paraffin sections and were shown to be absent at an external diagnostic laboratory (LSI Medience Corporation). The patient then, underwent intravenous administration of pembrolizumab $77.2 \mathrm{mg}$ every three weeks five times in total.

At three months after the conclusion of proton beam therapy, five and a half months from the initial visit, ocular surface melanoma almost subsided (Fig. 1D and E) and the clear cornea allowed visualization of the intraocular lens inside the eye (Fig. 1F). In three weeks, spontaneous corneal perforation was plugged with iris incarceration (Fig. 1G). Around the same time, the patient felt malaise and was found to have hypothyroidism which was evidenced by low free $\mathrm{T} 3$ at $1.75 \mathrm{pg} / \mathrm{ml}$, low free T4 at $0.90 \mathrm{ng} / \mathrm{dl}$, high TSH at $39.9 \mu \mathrm{U} / \mathrm{ml}$, high anti-thyroglobulin antibody $(\mathrm{TgAb})$ at $20,664 \mathrm{IU} / \mathrm{ml}$ and high anti-thyroid peroxidase antibody (TPOAb) at 2,580 IU/ml. The patient started to take levothyroxine sodium hydrate

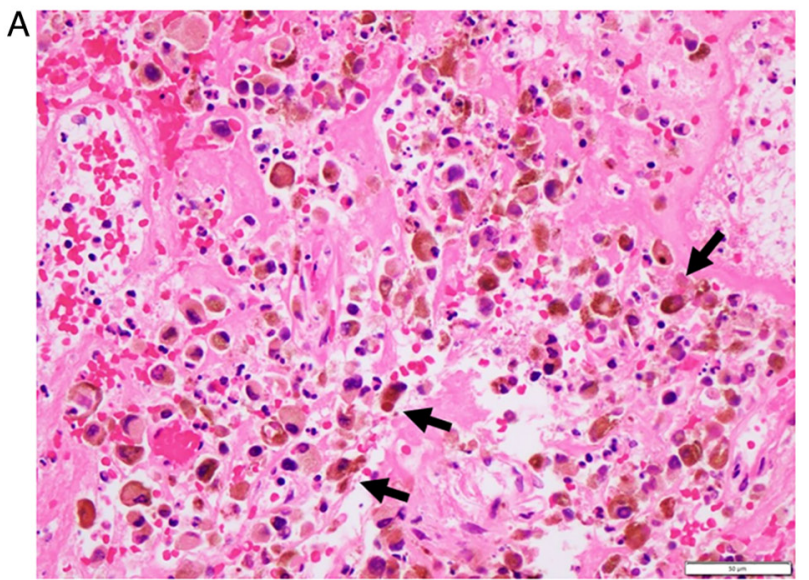

B

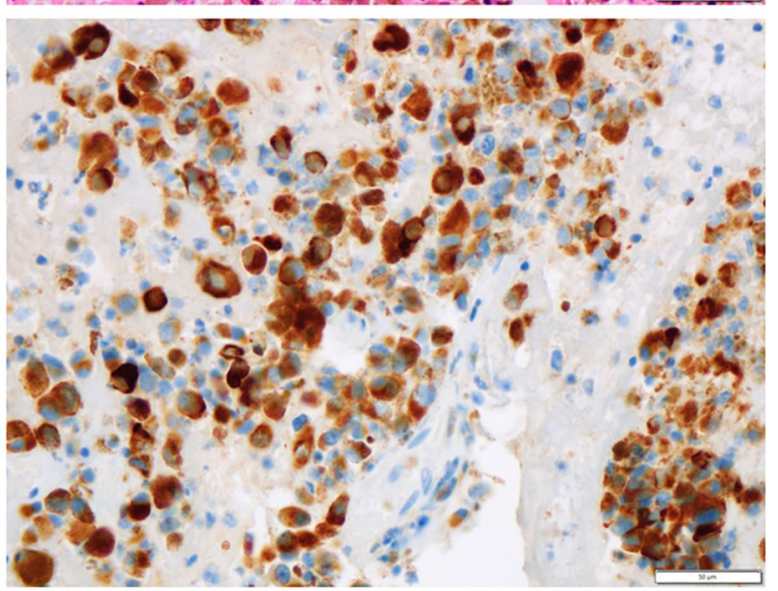

Figure 3. (A) Biopsy specimen in hematoxylin-eosin stain of the ocular surface mass at the initial visit. Note anomalous epithelioid cells (arrows) with melanin pigments which are dispersed in fibrin and hemorrhage. (B) The anomalous cells were positive for cocktail-mix antibodies against tyrosinase, melanoma antigen recognized by $\mathrm{T}$ cells- 1 and human melanoma black- 45 , indicative of malignant melanoma. Scale bar, $50 \mu \mathrm{m}$.

$0.25 \mu \mathrm{g}$ daily. She died suddenly of unknown cause 7.5 months from the initial visit.

\section{Discussion}

The clinical question in the present study is that proton beam therapy might be a treatment option in the standard of care for advanced conjunctival malignant melanoma in the era when proton beam therapy is included in the reimbursement of the national health insurance in Japan. Orbital exenteration was initially indicated for the local control of malignancy as the standard of care in this patient. However, the patient and her family wished to avoid radical surgery and the clinicians, also wished to search for the other options because of the patient's advanced age. Proton beam therapy was thus chosen as the first-line treatment for unresectable melanoma in the head and neck according to the rule of the national health insurance in Japan (16-18). The patient had satisfactory outcome and regained better quality of life: She had covered the left-side tumor with gauze eye patch for cosmetic reasons before the therapy while she could blink and close the eye with the eyelid on the left side after the therapy.

On the initial presentation, biopsy could only obtain superficial hemorrhage-prone tissue of the tumor and pathological 
A
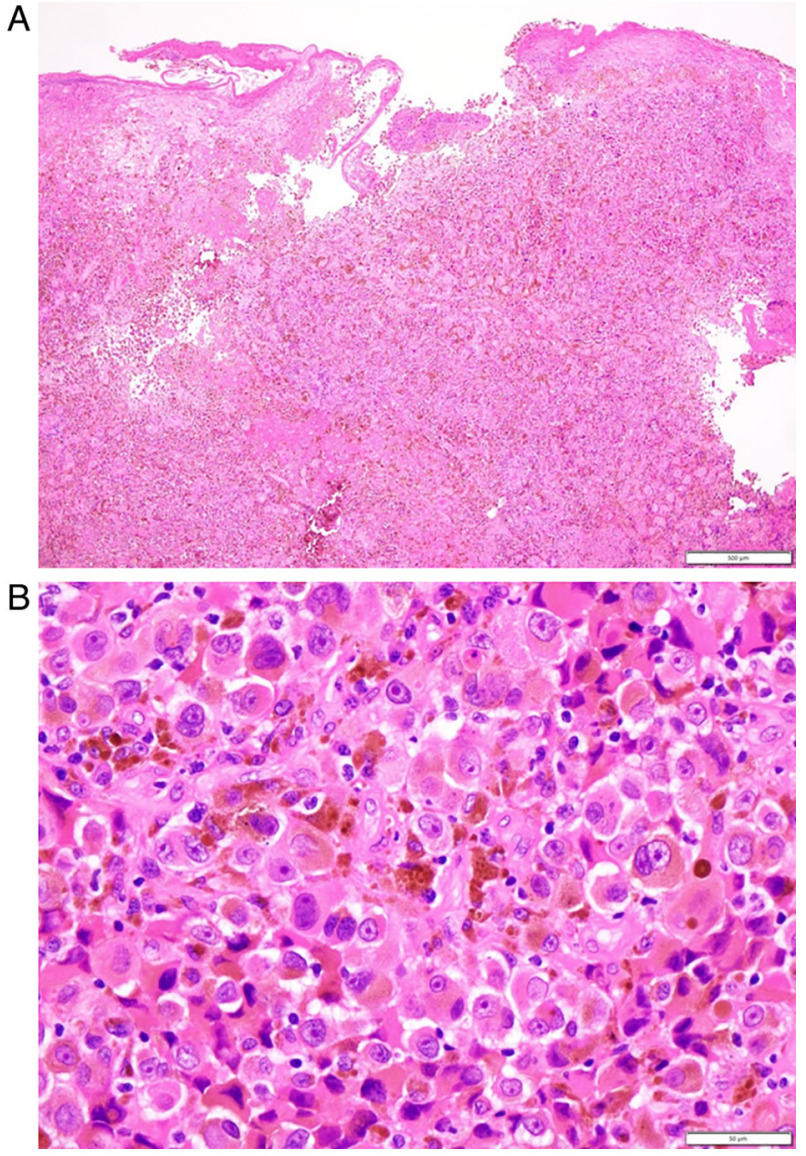

Figure 4. Biopsy of melanotic lesions in the upper eyelid skin on the left side one month after the conclusion of proton beam therapy, 3.5 months from the initial visit. Note melanin-containing epithelioid cells with large nuclei and apparent nucleoli in alveolar pattern, confirming pathological diagnosis of malignant melanoma. Hematoxylin-eosin stain. (A) Scale bar, $500 \mu \mathrm{m}$ and (B) $50 \mu \mathrm{m}$.

diagnosis was based on immunocytochemical examinations. Following the proton beam therapy, excisional biopsy of the residual eyelid lesion was done again to confirm the pathological diagnosis of malignant melanoma and also to examine $B R A F$ mutations in the melanoma tissue. Around the same time, the patient was found to have multiple metastatic lesions in bilateral lung fields. According to the standard protocol for unresectable and metastatic malignant melanoma $(19,20)$, pembrolizumab was introduced, instead of the combination of MEK inhibitor and BRAF inhibitor, based on the absence of the $B R A F$ mutation in the melanoma tissue. The sequence of events in this patient, namely, proton beam therapy for local control of unresectable conjunctival malignant melanoma, followed later by the administration of immune checkpoint inhibitors or the combination of MEK inhibitor and BRAF inhibitor, could become the standard of care in the modern era. Indeed, in the present patient, pembrolizumab appeared to have an effect on the reduction of malignant melanoma in the original and metastatic lesions, but actual evaluation of the effect could not be accomplished since the patient suddenly succumbed.

The patient was reported to have succumbed suddenly to unknown causes by the emergency transportation to a local hospital. The patient had hypothyroidism, probably induced by the administration of pembrolizumab, and thus was taking oral thyroid hormone. Autoimmune diseases, such as thyroiditis, pneumonitis and uveitis, should be kept in mind in patients who receive immune checkpoint inhibitors (21). The sudden mortality might be attributed to possible carditis or pneumonitis as a manifestation of pembrolizumab-induced autoimmune diseases, but the exact situation was not confirmed clinically in the patient. Until the sudden mortality, the patient was healthy and had normal activities of daily life.

A major limitation in this case report is that the patient's presentation was unusual and that the sequence of event was thus out of the standard. First, only the surface biopsy could be done in the large protruding hemorrhage-prone melanoma instead of standard resection. Secondly, proton beam therapy was chosen as the first-line treatment for this giant conjunctival melanoma. The aim of therapeutic strategy at the initial presentation was to enhance the quality of life in this aged woman and the corneal melt as a sequel to proton beam therapy was anticipated in advance. On the initial phase of the patient's presentation, it was planned to use immune checkpoint inhibitors or molecular target drugs as an adjunct therapy since distant metastases to lung or liver would be unavoidable at the stage of the disease. In this context, it was not expected the complete response to proton beam therapy alone and residual melanoma lesions in the eyelid skin could be used as the biopsy site for examining the $B R A F$ mutation to determine the appropriate adjunct therapy. It should be emphasized that the present case would not be recognized as the standard for local control of conjunctival melanoma at the current time.

In conclusion, proton beam therapy could be placed as a treatment option for local control of unresectable conjunctival malignant melanoma. Proton beam therapy as the first-line treatment, followed by adjunct therapy with immune checkpoint inhibitors, could become the standard of care in near future for unresectable giant conjunctival melanoma. This conclusion, based on a single patient, naturally has a limitation to being generalized to the other patients with conjunctival malignant melanoma.

\section{Acknowledgements}

Not applicable.

\section{Funding}

No funding was received.

\section{Availability of data and materials}

The datasets used and/or analyzed during the current study are available from the corresponding author on reasonable request.

\section{Authors' contributions}

TM, as an ophthalmologist and OY, as a dermatologist, followed and treated the patient, TT, as a pathologist, made the pathological diagnosis and $\mathrm{KK}$ and TW, as radiologists, treated the patient. TM wrote the manuscript and OY, TT, KK and TW did critical review of the manuscript. All authors 
confirm the authenticity of all the raw data and approved the final version of the manuscript.

\section{Ethics approval and consent to participate}

Ethics committee review was not applicable to case reports, based on the Ethical Guidelines for Medical and Health Research Involving Human Subjects, issued by the Government of Japan.

\section{Patient consent for publication}

Verbal informed consent was obtained from the patient for her anonymized information to be published in this article.

\section{Competing interests}

The authors declare that they have no competing interests

\section{References}

1. Matsuo T, Ogino Y, Ichimura K, Tanaka T and Kaji M: Clinicopathological correlation for the role of fluorodeoxyglucose positron emission tomography computed tomography in detection of choroidal malignant melanoma. Int J Clin Oncol 19: 230-239, 2014.

2. Matsuo T, Tanaka T and Yamasaki O: Lacrimal sac malignant melanoma in 15 Japanese patients: Case report and literature review. J Investig Med High Impact Case Rep 7: 2324709619888052, 2019.

3. Seregard S: Conjunctival melanoma. Surv Ophthalmol 42: 321-350, 1998.

4. Vora GK, Demirci H, Marr B and Mruthyunjaya P: Advances in the management of conjunctival melanoma. Surv Ophthalmol 62 26-42, 2017.

5. Jain P, Finger PT, Fili M, Damato B, Coupland SE, Heimann H, Kenawy N, Brouwer NJ, Marinkovic M, Van Duinen SG, et al: Conjunctival melanoma treatment outcomes in 288 patients: A multicentre international data-sharing study. Br J Ophthalmol 105 1358-1364, 2021.

6. Ballo MT and Ang KK: Radiotherapy for cutaneous malignant melanoma: Rationale and indications. Oncology (Williston Park) 18: 99-110, 113-114, 2004.

7. Gorayski P, Burmeister B and Foote M: Radiotherapy for cutaneous melanoma: Current and future applications. Future Oncol 11: 525-534, 2015.

8. López F, Rodrigo JP, Cardesa A, Triantafyllou A, Devaney KO, Mendenhall WM, Haigentz M Jr, Strojan P, Pellitteri PK, Bradford CR, et al: Update on primary head and neck mucosal melanoma. Head Neck 38: 147-155, 2016.

9. Grant-Freemantle MC, O'Neill BL and Clover AJP: The effectiveness of radiotherapy in the treatment of head and neck mucosal melanoma: Systematic review and meta-analysis. Head Neck 43: 323-333, 2021
10. Wuestemeyer H, Sauerwein W, Meller D, Chauvel P, Schueler A, Steuhl KP, Bornfeld N and Anastassiou G: Proton radiotherapy as an alternative to exenteration in the management of extended conjunctival melanoma. Graefes Arch Clin Exp Ophthalmol 244: 438-446, 2006

11. Westekemper H, Anastassiou G, Sauerwein W, Chauvel P, Bornfeld N, Steuhl KP and Meller D: Analysis of ocular surface alterations following proton beam radiation in eyes with conjunctival malignant melanoma. Ophthalmologe 103: 588-595, 2006 (In German).

12. Krause L, Mladenova A, Bechrakis NE, Kreusel KM, Plath T, Moser L and Foerster M: Treatment modalities for conjunctival melanoma. Klin Monbl Augenheilkd 226: 1012-1016, 2009 (In German).

13. Maschi-Cayla C, Doyen J, Gastaud P and Caujolle JP: Conjunctival melanomas and proton beam therapy. Acta Ophthalmol 91: e647, 2013.

14. Scholz SL, Hérault J, Stang A, Griewank KG, Meller D, Thariat J, Steuhl KP, Westekemper H and Sauerwein W: Proton radiotherapy in advanced malignant melanoma of the conjunctiva. Graefes Arch Clin Exp Ophthalmol 257: 1309-1318, 2019.

15. Thariat J, Salleron J, Maschi C, Fevrier E, Lassalle S, Gastaud L, Baillif S, Claren A, Baumard J, Herault J and Caujolle JP: Oncologic and visual outcomes after postoperative proton therapy of localized conjunctival melanomas. Radiation Oncol 14: 239, 2019.

16. Zenda S, Kawashima M, Nishio T, Kohno R, Nihei K, Onozawa M, Arahira S and Ogino T: Proton beam therapy as a nonsurgical approach to mucosal melanoma of the head and neck: A pilot study. Int J Radiat Oncol Biol Phys 81: 135-139, 2011.

17. Fuji H, Yoshikawa S, Kasami M, Murayama S, Onitsuka T, Kashiwagi $\mathrm{H}$ and Kiyohara Y: High-dose proton beam therapy for sinonasal mucosal malignant melanoma. Radiat Oncol 9: 162, 2014.

18. Sakurai H, Ishikawa $\mathrm{H}$ and Okumura T: Proton beam therapy in Japan: Current and future status. Jpn J Clin Oncol 46: 885-892, 2016.

19. Larsen AC, Dahmcke CM, Dahl C, Siersma VD, Toft PB, Coupland SE, Prause JU, Guldberg P and Heegaard S: A retrospective review of conjunctival melanoma: Presentation, treatment and outcome and an investigation of features associated with BRAF mutations. JAMA Ophthalmol 133: 1295-1303, 2015.

20. Kiyohara T, Tanimura H, Miyamoto M, Shijimaya $T$, Nagano N, Nakamaru S, Makimura K and Iwai H: Two cases of BRAF-mutated, bulbar conjunctival melanoma, and review of the published literature. Clin Exp Dermatol 45: 207-211, 2020.

21. Matsuo T and Yamasaki O: Vogt-Koyanagi-Harada disease-like posterior uveitis in the course of nivolumab (anti-PD-1 antibody), interposed by vemurafenib (BRAF inhibitor), for metastatic cutaneous malignant melanoma. Clin Case Rep 5: 694-700, 2017.

This work is licensed under a Creative Commons Attribution-NonCommercial-NoDerivatives 4.0 International (CC BY-NC-ND 4.0) License. 